\title{
A case of epidermoid median raphe cyst traversing the corpora
} cavernosa

\author{
Alice Yu, MD; John-Paul Capolicchio, MDCM, FRCSC, FAAP \\ Division of Pediatric Urology, Department of Pediatric Surgery, Montreal Children's Hospital, McGill University Health Centre, Montreal, QC, Canada
}

Cite as: Can Urol Assoc J 2017;11 (3-4):E1 19-21. http://dx.doi.org/10.5489/cuaj.4153 Published online March 16, 2017

\section{Abstract}

Median raphe cysts are congenital lesions that typically have a superficial appearance. We present a very unusual case of a deep perineal mass in a six-year-old boy. The lesion extends into the corpus cavernosum, suggesting that the anomaly was an early embryological event. Histopathological features are consistent with an epidermoid type of median raphe cyst.

\section{Case report}

A six-year-old otherwise healthy boy presented to the emergency room with a painless and rapidly enlarging mass in the scrotum and perineum. He denied urinary or gastrointestinal symptoms. The impression at that time was a neoplastic process, therefore ultrasound and magnetic resonance imaging (MRI) were performed.

The ultrasound reported a heterogeneous mass in the midline of the scrotum, between the tunica of the left and right testicle. Further evaluation by MRI showed a fluid-filled cystic structure in the midline of the scrotum with extension posteriorly to the base of the penis (Fig. 1).

During the evaluation, five days following initial presentation, the patient became febrile with leukocytosis $(14.4 \mathrm{x}$ $10^{9} / \mathrm{L}$ ). The mass became very tender and there was overlying erythema on physical exam. The differential diagnosis shifted from tumour to infection. He was brought to the operating room for surgical exploration. Upon bivalving the scrotum, a large amount of foul-smelling pus was evacuated under pressure. Culture of the pus grew coagulase-negative staphylococcus and mixed anaerobic flora. Biopsy of the cyst wall was sent to pathology, later reported as chronic inflammation with no evidence of malignancy. Intraoperative cystoscopy was performed, revealing no valve or diverticulum in the urethra and a normal bladder. The cystoscope was also inserted into the open abscess cavity, which was blind-ended with a one-inch diameter conical extension. The abscess appeared to be lying directly below the urethra, but without connecting.

At that time, the possibility of a fistula to the bowel was considered by the general surgical service consulted in the case. The wound was left open with packing and the patient was taken back to the operating room 72 hours later for re-exploration under general anesthesia. The wound was healing well, therefore he was discharged home on oral antibiotics and daily dressing changes. He also had a followup voiding cystourethrogram that was normal.

The patient came back four months later complaining of pus draining from a sinus tract in the scrotum. He was brought to the operating room for re-exploration and an abscess cavity was found and drained. A Penrose drain was left in. A repeat MRI was done and the images appeared similar to the original. The main differential diagnosis at this point was an infected Cowper's gland.

Two weeks later, he was taken back to the operating room for definitive management. A midline scrotal and perineal incision was made and extended from the middle of the scrotum down to the anus. The abscess was identified and was found tracking in a duct-like fashion towards the right corpora cavernosa. Further exploration revealed that the duct actually penetrated through the right cavernosum (Fig. 2).

Histology revealed epidermal cyst with keratinized squamous lining. At the five-year followup, the patient is doing well, with no clinical or ultrasound recurrence, nor any urinary or intestinal symptoms.

\section{Discussion}

Median raphe cysts are rare congenital structures that usually present in the midline of the perineum anywhere between the meatus to the anus. They have a typical superficial appearance and never communicate with the urethra. Although embryogenesis is unclear, two theories have been proposed. One suggests that the cysts arise from ectopic 
Yu et al.

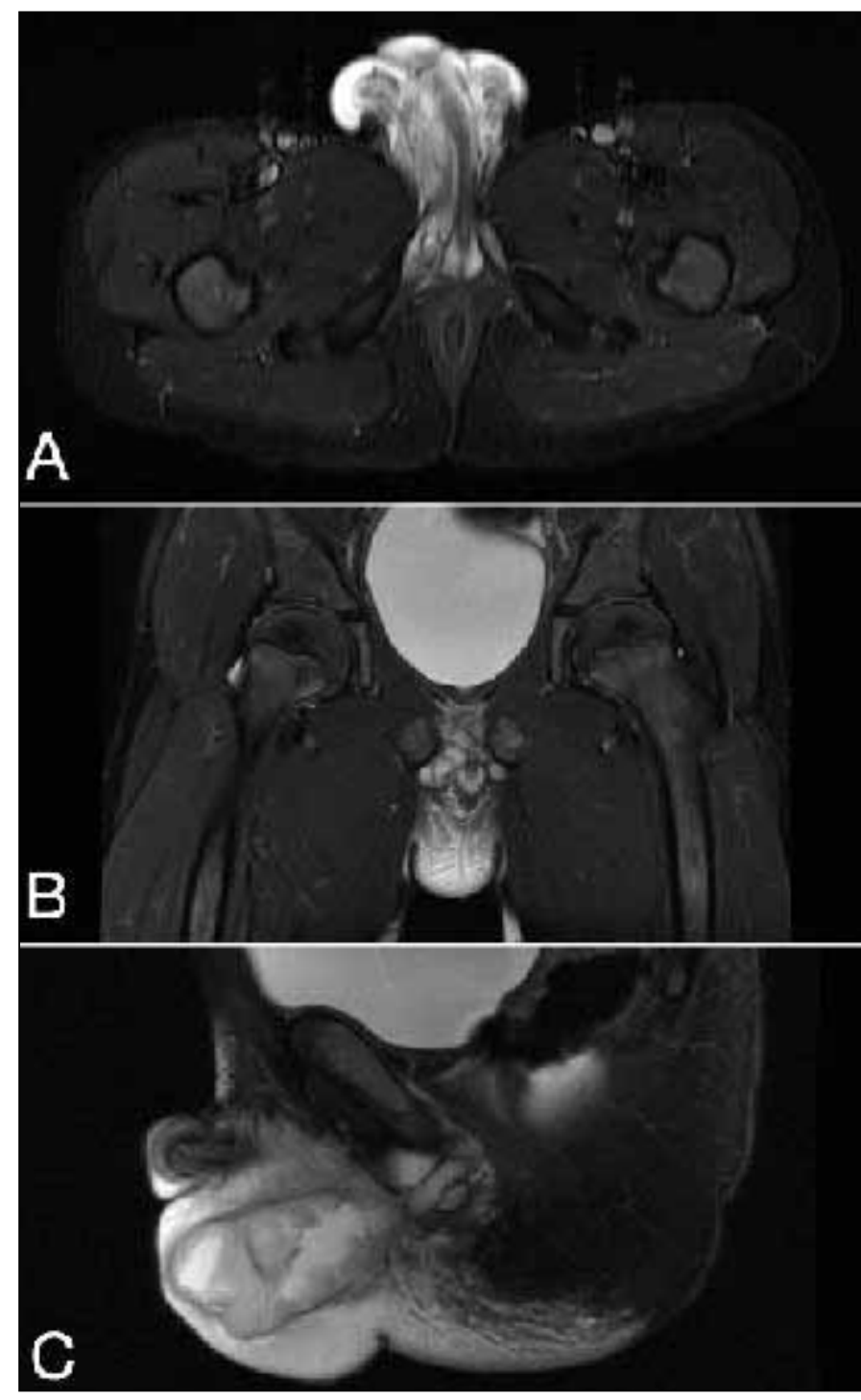

Fig. 1. Magnetic resonance imaging showing intrascrotal median raphe cystic lesion with extension into the base of the penis in various views: $(\boldsymbol{A})$ axial; $(\boldsymbol{B})$ coronal; and (C) sagittal.

urethral mucosa sequestered during embryological development. ${ }^{1}$ Another theory is that the cysts develop from abnormal closure of the median raphe and urethral groove. ${ }^{2}$

Histologically, there are three patterns: urethral type, lined with pseudostratified columnar epithelium; epidermoid type, lined with squamous stratified epithelium; and mixed type, which incorporates both types of epithelium. ${ }^{3}$ We suggest that our patient had an epidermoid type of median raphe cyst.

Median raphe cysts involving deeper structures in the perineum are extremely rare. We found two cases in the literature describing epidermoid cysts in the midline of the perineum with extension to the true pelvis. ${ }^{4,5}$ To our knowledge, this is the first case describing extension into the corpus cavernosum.

The passage of the duct through the corpus cavernosum has important implications, suggesting that the origin of the defect must be a very early embryological event. It would be very difficult for a benign lesion to penetrate tough tissue like the corpus cavernosum. The anomaly must have developed early in the closure of the median raphe at around 6-8 weeks of gestation.

One differential diagnosis was a syringocele, which was suspected based on the MRI and intraoperative findings. ${ }^{6}$ Others have also described unusual syringoceles in which the published histology was more in keeping with an epidermoid type median cyst. ${ }^{7}$

\section{Conclusion}

These cases suggest that even though median raphe cysts are typically superficial, they can also present deep in the perineum. It seems there is a spectrum of median raphe disorders. We hypothesize that the location of the lesion is dependent on when the defect occurs during closure of the median raphe early in gestation.

Competing interests: The authors report no competing personal or financial interests.

This paper has been peer-reviewed.

\section{References}

1. Cole LA, Helwig EB. Mucoid cysts of the penile skin. J Urol 1976;115:397-400.

2. Asarch RG, Golitz LE, Sausker WF, et al. Median raphe cysts of the penis. Arch Dermatol 1979; 115: 1084-6. https://doi.org/10.1001/archderm.1979.04010090034019

3. Krauel L, Tarrado X, Garcia-Aparicio L, et al. Median raphe cysts of the perineum in children. Urology 2008;71:830-1. hitps://doi.org/10.1016/j.urology.2007.11.131

4. Huang A, Palmer LS, Levitt SB. Epidermoid cyst of the scrotum extending into the true pelvis. Urology 1999;54:561. https://doi.org/10.1016/S0090-4295(99)00197-1

5. Sadler BT, Greenfield SP, Wan J, et al. Intrascrotal epidermoid cyst with extension into the pelvis. J Urol 1995;153:1265-6. https://doi.org/10.1016/S0022-5347(01)67583-9

6. Redman JF, Rountree GA. Pronounced dilatation of Cowper's gland duct manifest as a perineal mass: A recommendation for management. J Urol 1988;139:87-8.

7. Marte A, Prezioso M, Sabatino MD, et al. [Syringocele in children: An unusual presentation as scrotal mass]. Minerva Pediatr 2009;61:123-7.

Correspondence: Dr. J-P Capolicchio, Division of Pediatric Urology, Department of Pediatric Surgery, Montreal Children's Hospital, McGill University Health Centre, Montreal, QC, Canada; JP.Capolicchio@muhc.mcgill.ca 


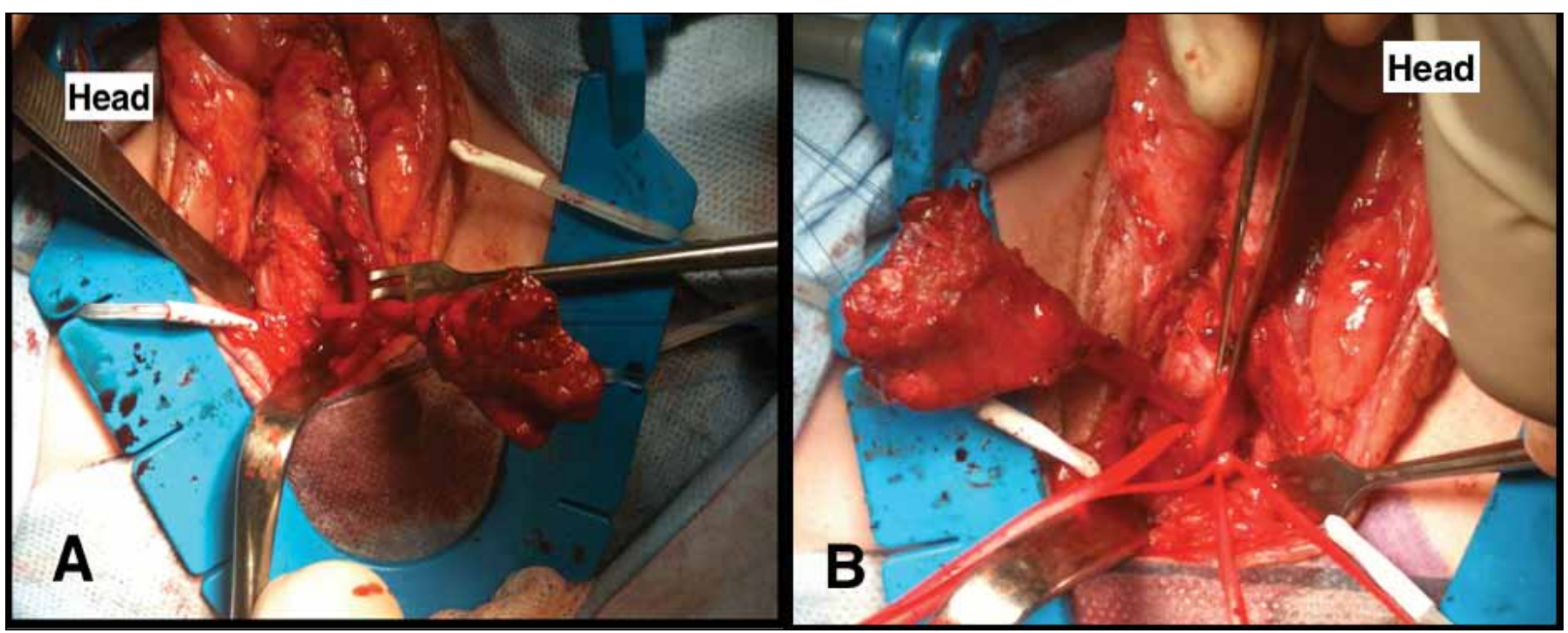

Fig. 2. (A) The duct connecting to the cystic lesion penetrates through the cavernosum tissue; and (B) right vessel loop traverses the right corpus cavernosum in a bloodless plane; left vessel loop surrounds the deep end of the stalk which penetrates dorsal to the corpus. 